\title{
Closed-Orbit Theory of Oscillations in Atomic Photoabsorption \\ Cross Sections in a Strongelectric Field. I. Comparison Between Theory and Experiments on Hydrogen and Sodium Above Threshold
}

J. Gao

William \& Mary

J. B. Delos

William \& Mary, jbdelos@wm.edu

M. Baruch

Follow this and additional works at: https://scholarworks.wm.edu/aspubs

Part of the Physics Commons

\section{Recommended Citation}

Gao, J.; Delos, J. B.; and Baruch, M., Closed-Orbit Theory of Oscillations in Atomic Photoabsorption Cross Sections in a Strongelectric Field. I. Comparison Between Theory and Experiments on Hydrogen and Sodium Above Threshold (1992). Physical Review A, 46(3), 1449-1454.

https://doi.org/10.1103/PhysRevA.46.1449

This Article is brought to you for free and open access by the Arts and Sciences at W\&M ScholarWorks. It has been accepted for inclusion in Arts \& Sciences Articles by an authorized administrator of W\&M ScholarWorks. For more information, please contact scholarworks@wm.edu. 


\title{
Closed-orbit theory of oscillations in atomic photoabsorption cross sections in a strong electric field. I. Comparison between theory and experiments on hydrogen and sodium above threshold
}

\author{
J. Gao and J. B. Delos \\ Physics Department, College of William and Mary, Williamsburg, Virginia 23187 \\ M. Baruch \\ Physics Department, University of Virginia, Charlottesville, Virginia 22901
}

(Received 3 December 1991)

\begin{abstract}
Using a simple analytic formula from closed-orbit theory, we calculate photoabsorption cross sections of hydrogen and sodium in a strong electric field. The theoretical spectra show good agreement with experimental results. A scaled variable measurement is also suggested.

PACS number(s): 32.60. $+\mathrm{i}, 32.80 . \mathrm{Cy}, 32.80 . \mathrm{Fb}$
\end{abstract}

\section{INTRODUCTION}

If an atom is placed in a constant electric field and the absorption spectrum is measured with photon energy above the zero-field ionization threshold, then the absorption spectrum as a function of energy shows oscillations. In the accompanying paper [1] we present a complete theoretical analysis of this phenomenon. Here we compare the resulting formulas with experiments.

The physical picture is based upon the closed-orbit theory given in Ref. [2]. When a laser is applied to an atom in an electric field, the atom may absorb a photon. When it does, the electron goes into a near-zero-energy outgoing Coulomb wave. This wave then propagates away from the nucleus. Sufficiently far from the nucleus, the wave propagates according to semiclassical mechanics, and it is correlated with classical trajectories. The wave fronts are perpendicular to the trajectories. Eventually, the trajectories and the wave fronts are turned back by the electric field, some of the orbits return to the vicinity of the nucleus, and the associated waves (now incoming) interfere with the outgoing waves to produce the observed oscillations in the absorption spectrum.

For an atom in an electric field, above the zero-field ionization energy $(E>0)$, there is only one closed orbit, which goes from the Coulomb center along the electric field and returns to the center. The oscillations in the spectrum arise from this orbit and its repetitions. This situation is simple enough that a closed-form expression can be given for the photoabsorption cross section [Eq. (2.3)]. For hydrogen, this formula was derived by Bogomol'nyi [3] (see also Ref. [4]). In the accompanying paper [1] we show that practically the same formula also describes photoabsorption for alkali-metal atoms. Only two differences between hydrogen and alkali metals appear: (1) The oscillations are very slightly displaced as a result of a phase shift $\Delta$, which is related to quantum defects of the alkali-metal atoms, and (2) the amplitude of the oscillations is substantially changed. Since the single closed orbit is along the electric field, for the hydrogen atom oscillations will exist if the electric field of the light is polarized parallel to the applied field ( $\pi$-polarized light). If the electric field of the light is perpendicular to the applied electric field ( $\sigma$-polarized light), oscillations are predicted not to exist (or to be very tiny). For alkalimetal atoms, on the other hand, the initial $n^{2} P_{3 / 2}$ and ${ }^{2} P_{1 / 2}$ states are superpositions of $Y_{l m}$ 's. This modifies the angular distribution of outgoing waves such that oscillations are visible for either polarization of the light.

The paper is organized as follows. (All theoretical details are presented in the accompanying paper.) In Sec. II of this paper, we quote the final result for the abovethreshold case, and specify the numerical values of the quantities appearing in the formula for hydrogen and sodium. In Sec. III we compare our calculated results with other theories $[5,6]$ and with experiments carried out a few years ago by Rottke and Welge [6] and Luk et al. [7], and with experimental results reported here. Finally, in Sec. IV we suggest a scaled-energy experiment, in which laser energy and electric field would be varied simultaneously. We display our calculated results and show that the use of scaled variables in data recording leads to an especially simple description.

\section{QUANTITATIVE FORMULA}

The rate of absorption of photons, or the rate of production of ions, is related to the photoionization cross section $\sigma(E, F)$ by

$$
\frac{d N}{d t}=I_{0} N_{i}\left(E-E_{i}\right)^{-1} \sigma(E, F),
$$

and the cross section is related to the oscillator-strength density $D f(E, F)$ by

$$
\sigma(E, F)=\frac{2 \pi^{2} e^{2}}{m_{e} c} D f(E, F) .
$$

In Ref. [1] it is shown that for energies above, but close to, the zero-field ionization threshold $E=0$, the oscillator-strength density can be written as a smooth "background" term plus a sum of sinusoidal oscillations, 


$$
\begin{aligned}
& D f(E, F)= D f_{0}(E)+g(E) F^{1 / 4} \\
& \times \sum_{n=1}^{\infty} \frac{\varepsilon^{1 / 2}}{\sinh [\sqrt{2 \varepsilon} n \tau(\varepsilon)]} \\
& \quad \times \sin \left\{n\left[\hat{S}(\varepsilon) F^{-1 / 4}-\pi\right]+\Delta\right\} .
\end{aligned}
$$

$D f_{0}$, the smooth background term, is equal to the oscillator-strength density that would be obtained in the absence of the electric field. The remaining terms are oscillatory functions of energy; each term in the sum over $n$ arises from one of the repetitions of the single closed orbit. The prefactor $g(E)$ and the phase shift $\Delta$ are given by

$$
\begin{aligned}
& g(E)=2^{9 / 2}\left(E-E_{i}\right) \pi\left|\widetilde{\mathscr{H}}_{0}(0)_{\mathscr{y}_{0}}(0)\right|, \\
& \Delta=\arg \left[\widetilde{\mathscr{Y}}_{0}(0)_{\mathscr{Y}_{0}}(0)\right] .
\end{aligned}
$$

The quantity $\mathscr{y}_{0}(\theta)$ is defined in Eqs. (5.12), (5.14), (5.17), and (5.18) of Ref. [1]. It represents the angular distribution of the initial outgoing electron waves that are produced by photoionization. $\mathscr{y}_{0}(0)$ is the amplitude of the wave at $\theta=0$, the direction of the closed orbit. This quantity is proportional to the radial dipole integrals between bound and free states, $I\left(n, l, l^{\prime}\right)$. These integrals are smoothly varying functions of electron energy, and they can be approximated by constants over the energy range of our calculation.

$\varepsilon$ is the scaled energy $\varepsilon=E / F^{1 / 2} . \tau(\varepsilon)$ is the scaled period of the closed orbit and $\widehat{S}(\varepsilon)$ is the scaled action. $\tau$ and $\hat{S}$ are classical quantities calculated along the closed orbit, and they can be expressed by elliptic integrals,

$$
\begin{aligned}
& \tau(\varepsilon)=\oint\left(4+2 \varepsilon u^{2}-u^{4}\right)^{-1 / 2} d u, \\
& \widehat{S}(\varepsilon)=\oint\left(4+2 \varepsilon u^{2}-u^{4}\right)^{1 / 2} d u
\end{aligned}
$$

Finally, the summation index $n$ labels the repetitions of the closed orbit. The phase $n\left[\hat{S}(\varepsilon) F^{-1 / 4}-\pi\right]$ is the action associated with $n$ traversals of the orbit, plus additional phase shifts, one arising from a caustic at the end point of the orbit and another from the Coulomb singularity. The expression

$$
F^{1 / 4} \varepsilon^{1 / 2} / \sinh [\sqrt{2 \varepsilon} n \tau(\varepsilon)]
$$

is proportional to the amplitude of the returning wave on the $n$th return. Since the orbit is unstable, the amplitude decreases exponentially with $n$ for large $n$.

Relevant values of the constants needed for various transitions are summarized in Table I. Pseudo-H is hydrogen in which Stark mixing of $s, p$, and $d$ terms in the initial state is ignored, and the initial state is imagined to be simply $3 p_{z}$.

\section{CALCULATION OF ABSORPTION SPECTRA}

We have calculated oscillations in the absorption spectrum for either hydrogen or sodium atoms in an electric field. We have compared the results of the above analytic formula with theoretical calculations by Rottke and Welge [6] on hydrogen, and with calculations by Harmin [5] on pseudo-H and $\mathrm{Na}$. In addition, we have compared our results with experimental measurements. Earlier experiments consist of measurements on hydrogen by Rottke and Welge [6] and measurements on sodium by Luk et al. [7] and by Sandner, Safinya, and Gallagher [8]. We found some discrepancies between our formula and the results obtained in Refs. [7] and [8]. Therefore, additional measurements on sodium have been made. We will show that agreement between our theory and these measurements is very respectable.

In making comparison, we define the modulation of the spectrum, $M$, as the difference between the first two adjacent extrema at $E \gtrsim 0$ divided by the smooth background. The energy spacing $\Delta E$ is defined as the energy difference between the first two peaks at $E \gtrsim 0$.

\section{A. Ionization spectrum for hydrogen}

We consider a field strength $F=5714 \mathrm{~V} / \mathrm{cm}$, and we assume that the electron was first excited to the $n=2 \mathrm{lev}$ el and then ionized. The external electric field couples $s$ and $p$ states, leading to a linear Stark effect (spin-orbit coupling is negligible). The initial-state wave function is

$$
\psi_{i}=\sqrt{\frac{1}{2}}\left(R_{20} Y_{00}+\lambda R_{21} Y_{10}\right) \text {, }
$$

where $\lambda=1(2 s+2 p$ state $)$ or $\lambda=-1(2 s-2 p$ state $)$. According to the selection rules, $\pi$-polarized light leads to transitions

$$
(2 s \pm 2 p)_{m=0} \longrightarrow(n s, n p, n d)_{m=0}, \quad E \approx 0 .
$$

The no-field cross sections for the two initial states are equal. Since the phase shift $\Delta$ is zero for hydrogen, each term in the sum of Eq. (2.3) depends on the property of the closed orbit only. Therefore, the difference between the two cross sections is due to the difference between the prefactors $g(E)$. The ratio of these factors for the two states is

$$
g_{2 s-2 p} / g_{2 s+2 p}=25,
$$

so the depth of the oscillation for ionization from the $2 s-2 p$ initial state is 25 times greater than that for ionization from the $2 s+2 p$ initial state.

Figure 1 shows the calculated cross section $\sigma_{2 s-2 p}$ at

TABLE I. Values of the characteristic constants. All quantities are in atomic units.

\begin{tabular}{lccccc}
\hline & Initial state & $D f_{0}$ & $\sigma_{0}$ & $g$ & $\Delta$ \\
\hline $\mathrm{H}$ & $2 s-2 p$ & 3.939 & 0.5675 & $15.46 \pi$ & 0 \\
$\mathrm{H}$ & $2 s+2 p$ & 3.939 & 0.5675 & $0.6184 \pi$ & 0 \\
Pseudo-H & $3 p_{z}$ & 8.356 & 1.203 & $22.48 \pi$ & 0 \\
$\mathrm{Na}$ & $3 p_{3 / 2}$ & 2.373 & 0.3420 & $4.082 \pi$ & 0.5184 \\
$\mathrm{Na}$ & $3 p_{1 / 2}$ & 1.936 & 0.2790 & $2.041 \pi$ & 0.5184 \\
\hline
\end{tabular}




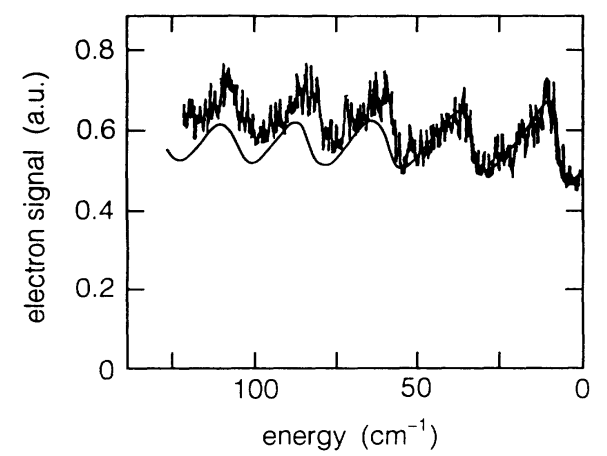

FIG. 1. Calculated photoionization cross section $\sigma_{2 s-2 p}$ of hydrogen in an electric field $F=5714 \mathrm{~V} / \mathrm{cm}$ with $\pi$-polarized light. The thin line is theory; the thick line is experiment (Ref. [5]).

$F=5714 \mathrm{~V} / \mathrm{cm}$. The modulation of $\sigma_{2 s-2 p}(E)$ (thin line) is $37.2 \%$, which lies in the experimental range $39 \pm 5 \%$ (thick line). The energy spacing is $27.85 / \mathrm{cm}$, in agreement with the experimental data. Our calculation also matches the theoretical calculation displayed in Ref. [6].

Our theory also exhibits further details of the oscillatory structure. Near $E=0$, the spectrum has a sawtooth shape, but as the energy increases, the oscillations become more symmetric and approach a sine wave. This is because only the $n=1$ term in Eq. (2.3) contributes significantly at high energy, but several terms contribute at low energy; repeated traversals of the orbit produce interference.

\section{B. Ionization spectrum for pseudohydrogen}

In order to make a comparison between ionization of hydrogen and ionization of alkali-metal atoms, Harmin used the initial state $3 p_{z}$ to calculate the hydrogen absorption spectrum in an electric field $F=77 \mathrm{kV} / \mathrm{cm}$. In other words, he ignored the linear Stark effect in hydro-

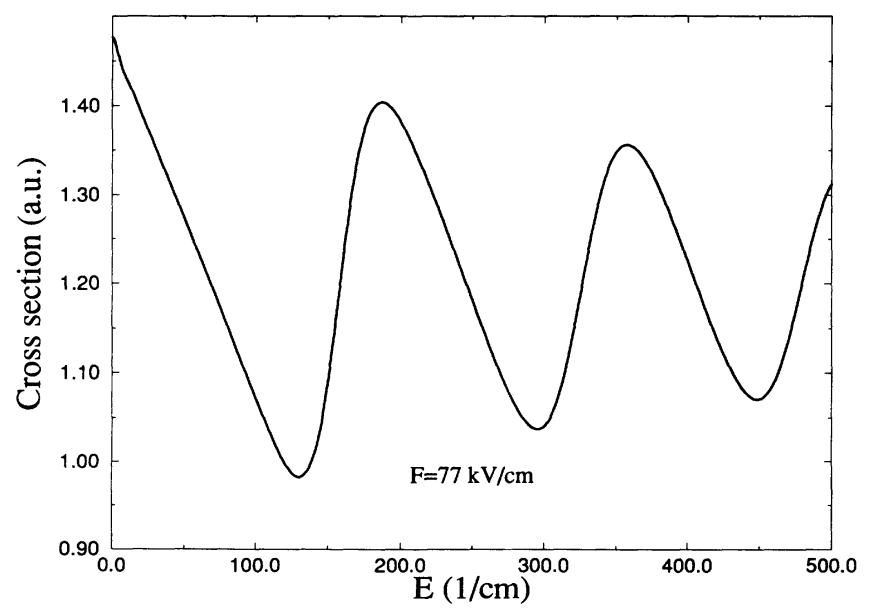

FIG. 2. Calculated photoionization cross section for pseudohydrogen at $F=77 \mathrm{kV} / \mathrm{cm}$ ( $\pi$ polarized). gen because it does not exist in alkali-metal atoms.

The initial-state wave function is

$$
\psi_{i}=R_{31} Y_{10},
$$

and the possible transitions are

$$
3 p_{m=0} \longrightarrow(n s, n d)_{m=0}, \quad E \approx 0 .
$$

Figure 2 shows the calculated spectrum, with modulation of $41 \%$ and energy spacing of $186.5 \mathrm{~cm}^{-1}$. Our spectrum generally agrees with Harmin's calculation, except in one respect. We have made the approximation that the zero-field ionization cross section is independent of energy; actually, it drifts downward somewhat as energy increases. Therefore, our oscillations agree with Harmin, but our background is about $5 \%$ too large at 250 $\mathrm{cm}^{-1}$.

\section{Ionization spectrum of sodium}

Comparison between the present theory and early experiments $[7,8]$ showed some discrepancies, so additional measurements are reported here. The experiment was performed using a two-step laser excitation scheme. Ground-state $\mathrm{Na}$ atoms were first excited to the $3 p$ state which has a large spin-orbit coupling $\left(17 \mathrm{~cm}^{-1}\right)$, far exceeding the laser linewidth $\left(1 \mathrm{~cm}^{-1}\right)$. Observations centered on the $3{ }^{2} P_{3 / 2}$ level; calculations have been made for both ${ }^{2} P_{3 / 2}$ and ${ }^{2} P_{1 / 2}$. This state was then further excited with a second laser to energies near the zero-field ionization threshold. In order to calibrate the second laser, a pickoff was directed through a Fabry-Pérot étalon with a nominal free spectral range of $6.66 \mathrm{~cm}^{-1}$. Using the well-known energies of the $\mathrm{Na}$ zero-field $s$ and $d$ states, $n=18$ through 28 , the étalon's actual spectral range was measured to be $6.433 \mathrm{~cm}^{-1}$. While recording the étalon fringes, the second laser was scanned in zero field continuously from an energy corresponding to $n=18$ and to an energy about $200 \mathrm{~cm}^{-1}$ above the zerofield ionization threshold, the highest recorded in the subsequent measurements. Provided the étalon's setup was not disturbed, the above-threshold energy range was therefore "linked" to the Na zero-field spectrum. Subsequent records of the étalon fringes, obtained for every laser scan with an electric field, were matched to the zero-field scan to check the validity of the calibration.

The electric field was provided by two parallel plates, $16 \times 16 \mathrm{~cm}^{2}$, separated by $1.5868 \mathrm{~cm}$. The error associated with the separation is at most $0.32 \%$. The voltage across the field plates was measured using a HewlettPackard high-voltage probe, which was calibrated using an HP digital voltmeter.

The initial state is then either

$$
R_{31}\left[\sqrt{\frac{2}{3}} Y_{10} \alpha+\sqrt{\frac{1}{3}} Y_{11} \beta\right], j=\frac{3}{2}, m_{j}=\frac{1}{2}
$$

or the alternative initial state (if the $j=\frac{1}{2}$ level had been selected)

$$
R_{31}\left[\sqrt{\frac{1}{3}} Y_{10} \alpha-\sqrt{\frac{2}{3}} Y_{11} \beta\right], \quad j=\frac{1}{2}, m_{j}=\frac{1}{2} .
$$

The analogous states having $m_{j}=-\frac{1}{2}$ are also equally populated. The transitions obey $\Delta l= \pm 1$ and $\Delta m_{l}=0$, 
and the spectrum is the superposition of two sets of transitions:

$$
\begin{aligned}
& \sigma_{3 / 2}=\frac{2}{3} \sigma_{m=0}+\frac{1}{3} \sigma_{m=1}, \\
& \sigma_{1 / 2}=\frac{1}{3} \sigma_{m=0}+\frac{2}{3} \sigma_{m=1} .
\end{aligned}
$$

Both $\Delta m=0$ and $\Delta m=1$ transitions contribute to the background, but the oscillations are caused by the $m=0$ final state only. This is because the closed orbit and its associated waves travel along the $z$ axis and only $m=0$ can produce an outgoing wave in that direction. The oscillatory terms in the cross sections for the two initial states differ by a factor of 2 , and the no-field cross sections differ by the ratio of

$$
\left(\sigma_{0}\right)_{2 / 3} /\left(\sigma_{0}\right)_{1 / 2}=1.22 \text {. }
$$

Therefore, the theory gives the modulation ratio for the two spectra:

$$
\frac{M_{1 / 2}}{M_{3 / 2}}=\frac{1}{2} \frac{\left(\sigma_{0}\right)_{3 / 2}}{\left(\sigma_{0}\right)_{1 / 2}}=0.61 \text {. }
$$
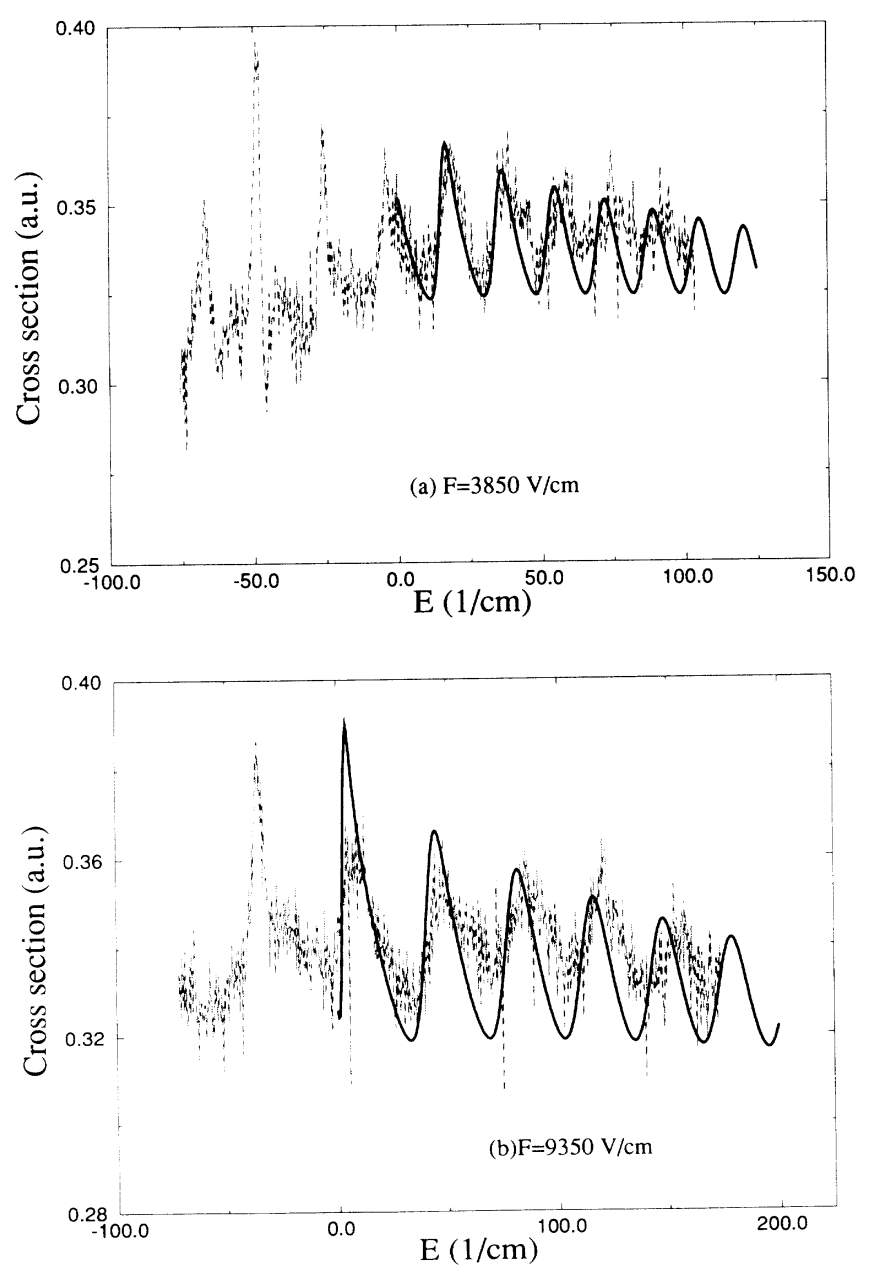

FIG. 3. Photoionization cross section of sodium with $3{ }^{2} P_{3 / 2}$ initial state at two different field strengths: (a) $F=3850 \mathrm{~V} / \mathrm{cm}$ and (b) $F=9530 \mathrm{~V} / \mathrm{cm}$. Dashed lines, new experiments; solid lines, theory.
The photoionization cross sections of sodium with the $3^{2} P_{3 / 2}$ initial state at two different electric-field strengths are shown in Fig. 3. The dashed lines represent our experimental data. The solid lines are the calculation. The agreement between the theory and the experiment is very good (though at high energy, experimental peaks have drifted a little away from theoretical ones) [9]. The theory shows that at $F=3850 \mathrm{~V} / \mathrm{cm}$, the spacing and modulation are $M=12.7 \%$ and $\Delta E=19.0 / \mathrm{cm}$, and both of them increase when $F$ increases. At $F=9350 \mathrm{~V} / \mathrm{cm}$, we have $M=20.8 \%$ and $\Delta E=41.5 / \mathrm{cm}$.

In an earlier measurement, Luk et al. reported the experimental results [10] for $\mathrm{Na}$ in a field strength $F=3590$ $\mathrm{V} / \mathrm{cm}$. Our calculated spacing $(19.5 / \mathrm{cm})$ and phase of the oscillations agree with experimental results, whereas the calculated modulation of the $3^{2} P_{3 / 2}$ initial state is $13.58 \%$, which exceeds the experimental value $(6 \pm 2) \%$. However, our results are close to Harmin's theoretical calculation on sodium (energy spacing, $19.6 \mathrm{~cm}^{-1}$, and modulation, $11 \%$ ).

\section{SCALED ENERGY SPECTROSCOPY}

The Hamiltonian of a hydrogen atom in a homogeneous static electric field is (taking $l_{z}=0$ )

$$
H=\left(\frac{1}{2}\right)\left(p_{z}^{2}+p_{\rho}^{2}\right)-1 /\left(\rho^{2}+z^{2}\right)^{1 / 2}+F z .
$$

The parameter $F$ can be eliminated using the scaling transformation

$$
\begin{aligned}
& \hat{\rho}=\rho / F^{1 / 2}, \quad \hat{z}=z / F^{1 / 2}, \\
& \hat{p}_{\rho}=p_{\rho} / F^{1 / 4} \quad \hat{p}_{z}=p_{z} / F^{1 / 4}, \quad \hat{t}=t F^{3 / 4}, \\
& \hat{H}=H / F^{1 / 2}=\left(\hat{p}_{z}^{2}+\hat{p}_{\rho}^{2}\right) / 2-1 /\left(\hat{\rho}^{2}+\hat{z}^{2}\right)^{1 / 2}+\hat{z} .
\end{aligned}
$$

It follows that all properties of the classical orbits do not depend on the energy $E$ and field strength $F$ independently, but only on the scaled energy $\varepsilon=E / F^{1 / 2}$. Hence, instead of studying the photoabsorption spectrum as a function of energy (at fixed $F$ ) and the Fourier transformation spectra in the time domain, it is more powerful to study the spectra with a fixed value of the scaled energy $\varepsilon=E / F^{1 / 2}$ and its Fourier transformation. One such measurement was carried out by Eichmann et al. [11].

The spectrum is studied at fixed scaled energy as a function of $F^{-1 / 4}$. We define

$$
\begin{aligned}
D f^{\prime} & =\frac{D f-D f_{0}}{E-E_{i}} F^{-1 / 4} \\
& =\sum_{n=1}^{\infty} C_{n} \sin \left\{n\left[\hat{S}(\varepsilon) F^{-1 / 4}-\pi\right]+\Delta\right\},
\end{aligned}
$$

where the amplitude is

$$
C_{n}=g^{\prime}(E) \frac{\sqrt{\varepsilon}}{\sinh [\sqrt{2 \varepsilon} n \tau(\varepsilon)]}, \quad g^{\prime}(E)=\frac{g(E)}{\left(E-E_{i}\right)}
$$

Over a small range of energy $g^{\prime}(E)$ is nearly constant. This equation says that if the absorption is measured at 
fixed scaled energy and if $D f^{\prime}$ is plotted versus $w=F^{-1 / 4}$, it becomes a superposition of perfect sine waves. Figures $4(a)$ and 4 (b) show the hydrogen spectrum at fixed scaled energies $\varepsilon=0.02$ and $\varepsilon=1$, respectively. As before, when $\varepsilon$ is small, the spectrum displays a sawtooth shape, and as $\varepsilon$ increases, the spectrum is more sinusoidal.

The Fourier-transformed spectra are no longer in the time domain, but are functions of a variable conjugate to $w=F^{-1 / 4}$, which we call $\mathcal{S}$,

$$
\begin{aligned}
\widetilde{D} f^{\prime}(\mathcal{S}) & =\frac{2}{w_{2}-w_{1}} \sum_{n=1}^{\infty} C_{n} \int_{w_{1}}^{w_{2}} \sin \{n[\hat{S}(\varepsilon) w-\pi]+\Delta\} e^{-i \mathcal{S} w} d w \\
& =\frac{2}{w_{2}-w_{1}} \sum_{n=1}^{\infty} \frac{C_{n}}{2 i}\left[e^{-i(n \pi-\Delta)} e^{i(n \hat{S}-\mathcal{S})\left(w_{2}-w_{1}\right)} /(n \hat{S}-\mathcal{S})+e^{i(n \pi-\Delta)} e^{-i(n \hat{S}+\mathcal{S})\left(w_{2}-w_{1}\right)} /(n \hat{S}+\mathcal{S})\right]
\end{aligned}
$$

Since $n \hat{S}>0$, the second sum can be neglected. The absolute square of the first sum gives the power spectrum of the oscillations

$$
\left|\widetilde{D} f^{\prime}(\mathscr{S})\right|^{2}=\sum_{j} C_{j}^{2} \frac{\sin ^{2}\left(a x_{j}\right)}{\left(a x_{j}\right)^{2}}+2 \sum_{j} \sum_{k<j} C_{j} C_{k} \cos \left[b\left(x_{j}-x_{k}\right)-\pi(j-k)\right] \frac{\sin \left(a x_{j}\right)}{\left(a x_{j}\right)} \frac{\sin \left(a x_{k}\right)}{\left(a x_{k}\right)},
$$
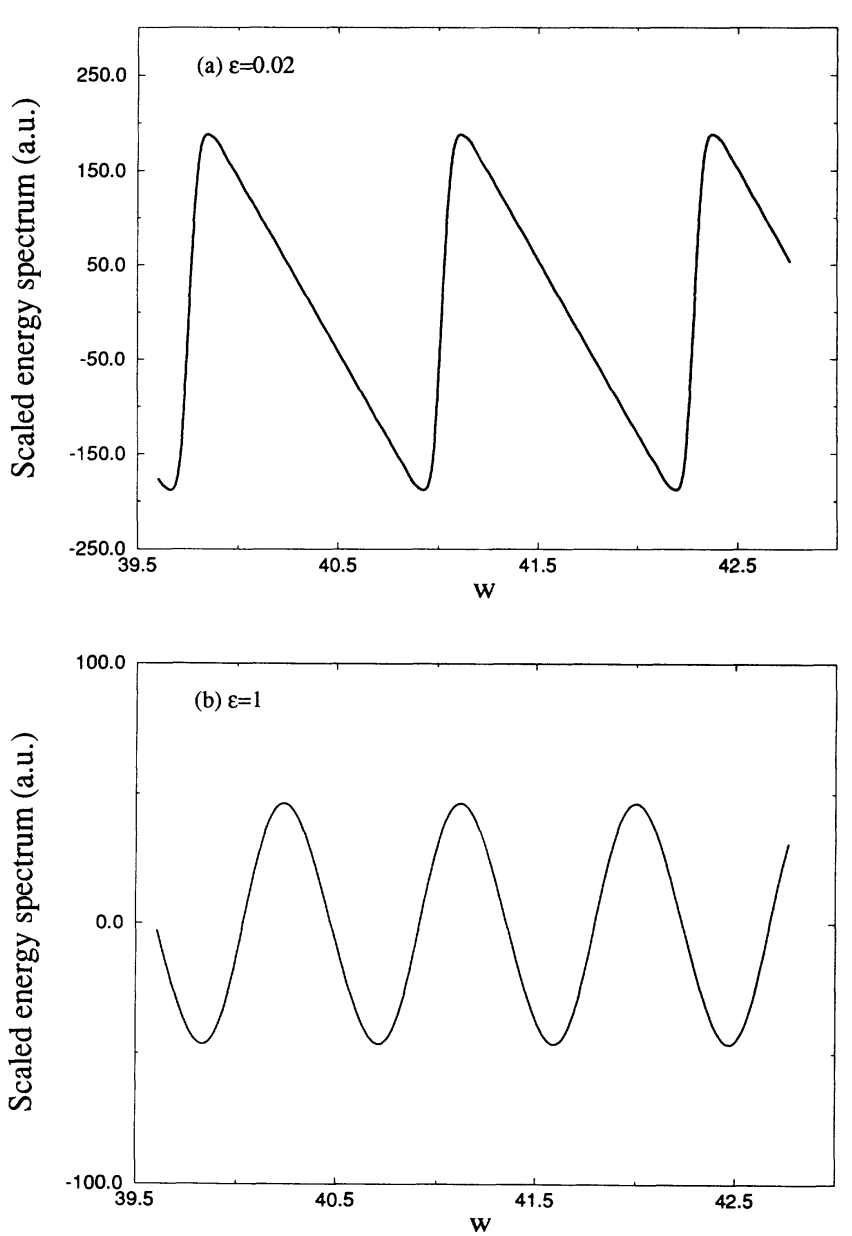

FIG. 4. Scaled-energy spectra for hydrogen at (a) $\varepsilon=0.02$ and (b) $\varepsilon=1.0$. The spectra cover the energy range from 2.4 to $2.8 \mathrm{~cm}^{-1}$ for (a) and 120 to $140 \mathrm{~cm}^{-1}$ for (b). In both cases the fields are varied from 1537.3 to $2092.4 \mathrm{~V} / \mathrm{cm}$ to keep the scaled energies constant. $w$ is dimensionless and is numerically equal to [electric field] $]^{-1 / 4}$ when the latter is measured in atomic units.
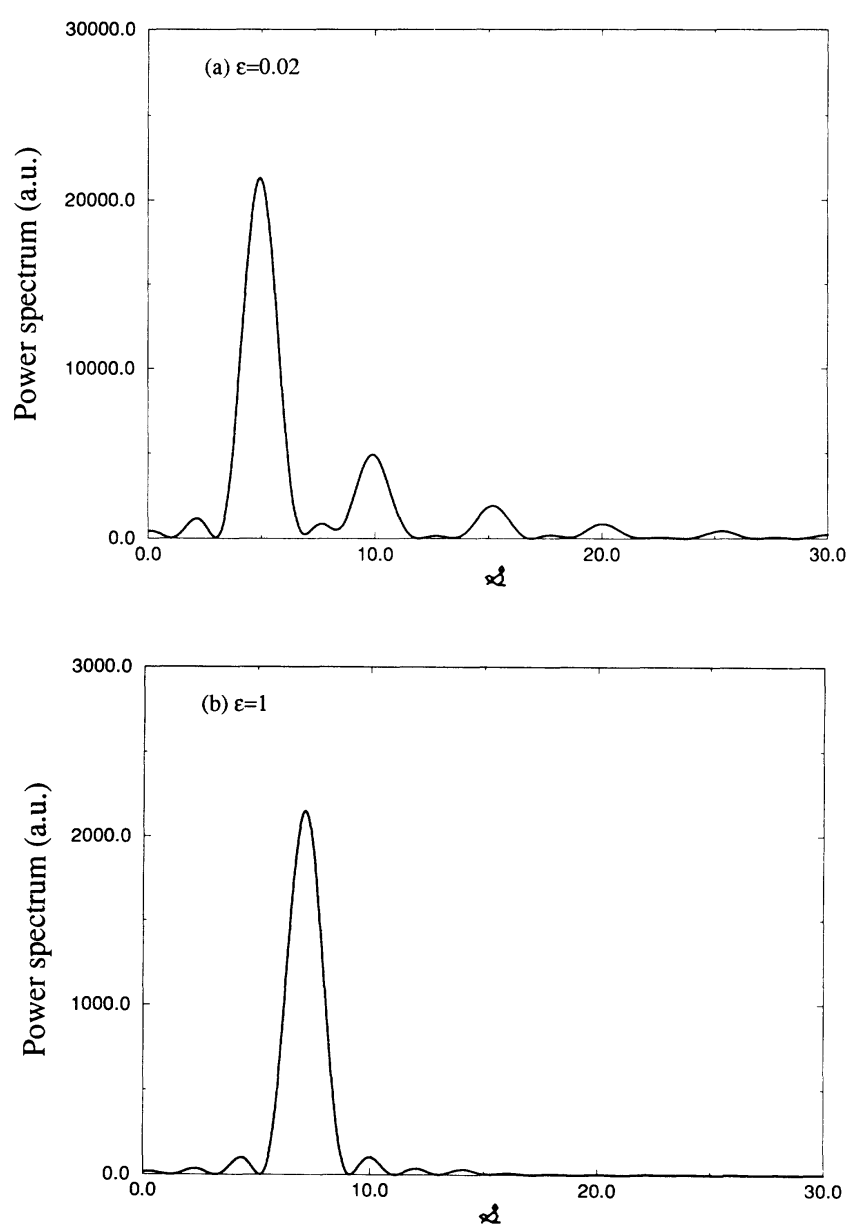

FIG. 5. Recurrence spectra of hydrogen at (a) $\varepsilon=0.02$ and (b) $\varepsilon=1.0$. The energy ranges and the field ranges are same as in Fig. 4. $\mathcal{S}$ is dimensionless and is numerically equal to [action times (electric field $)^{1 / 4}$ ], with these quantities measured in atomic units. The "atomic unit" for the power spectrum is (hartrees) $)^{-4}$. 
in which

$$
j \hat{S}-\mathcal{S}=x_{j}, \quad\left(w_{2}-w_{1}\right) / 2=a, \quad\left(w_{2}+w_{1}\right) / 2=b .
$$

The first term represents individual peaks associated with each repetition of the closed orbit, while the second term represents interference between those peaks if they overlap. (In the present case the overlap is small or negligible.) We call $\left|\widetilde{D} f^{\prime}\right|^{2}$ the "recurrence spectrum."

Figures 5(a) and 5(b) show the absolute square of the Fourier transform of the oscillatory part of the scaledvariable absorption spectrum, $\left|\widetilde{D} f^{\prime}\right|^{2}$ (the recurrence spectrum), with fixed scaled energies $\varepsilon=0.02$ and 1 , respectively. The spectrum has a very simple structure in which each peak in the Fourier transform corresponds to a repetition of the closed orbit. The positions of peaks are given by $\mathfrak{S}=n \hat{S}$, and the height of the peak is the oscillation amplitude $\left|C_{n}\right|^{2}$. The dominant modulation in the original cross section corresponds to the first peak in the Fourier transformation, i.e., the one at $\hat{S}$. The re- currence spectrum also indicates that the repetitions of the closed orbit have little effect except at $\varepsilon$ near zero.

The recurrence spectrum of sodium coincides with that of hydrogen apart from a normalization factor. The phase shift $\Delta$ of the $j$ th term cancels out with that of the $k$ th term.

Below threshold, the scaled-variable spectrum is more interesting. We plan to discuss it in a future paper.

\section{CONCLUSION}

A simple formula (2.3) describes the oscillations seen in the photoionization cross section of atoms above the zero-field ionization threshold.

\section{ACKNOWLEDGMENTS}

This research was supported by the National Science Foundation, the Office of Naval Research, the Jeffress Foundation, and the Air Force Office of Scientific Research. We thank Thomas Gallagher for many conversations and suggestions.
[1] J. Gao and J. B. Delos, following paper, Phys. Rev. A 46, 1455 (1992).

[2] M. L. Du and J. B. Delos, Phys. Rev. A 38, 1896 (1988); 38, 1913 (1988).

[3] E. B. Bogomol'nyi, Zh. Eksp. Teor. Fiz. 96, 487 (1989).

[4] A preliminary attempt at applying the ideas of periodicorbit theory to hydrogen in an electric field was made by Dieter Wintgen [J. Phys. B 22, L5 (1989)]. He recognized that a sum like our Eq. (2.3) must be relevant, but he did not obtain the magnitude of the oscillations relative to the background. In fact, since he focused on the density of states, that quantity cannot be obtained: The background density of states is infinite, but the oscillatory contribution is finite. What is needed is a formula for the photoabsorption cross section or the oscillator-strength density [1-3].

[5] David A. Harmin, Phys. Rev. A 26, 2656 (1982).

[6] H. Rottke and K. H. Welge, Phys. Rev. A 33, 301 (1986).

[7] T. S. Luk, L. Dimauro, T. Bergeman, and H. Metcalf, Phys. Rev. Lett. 47, 83 (1981).

[8] W. Sandner, K. A. Safinya, and T. F. Gallagher, Phys.
Rev. A 23, 2448 (1981).

[9] In this experiment, no wavelength standard was available at the high end of the energy range. As described in the text, the wavelength and photon energies were obtained by calibrating the étalon and extrapolating from standards approximately $30 \mathrm{~cm}^{-1}$ below threshold. A $5 \%$ error in this extrapolation could explain the discrepancy between theory and experiment at high energies. The formula (2.3) might be useful to calibrate energies or electric-field strengths when other standards are not available.

[10] Figure 2 of Ref. [7] incorrectly marked the zero-energy ionization threshold, $24473 \mathrm{~cm}^{-1}$, at approximately $24476 \mathrm{~cm}^{-1}$. The energy scale of the figure was also slightly nonlinear. T. Bergeman kindly sent an improved version of the figure, and our calculations give agreement with the wavelength and phase of the oscillations, but not with the amplitude.

[11] U. Eichmann, K. Richter, D. Wintgen, and W. Sandner, Phys. Rev. Lett. 61, 21 (1988). 